\title{
Efficacy and Safety of Evolocumab in Reducing Lipids and Cardiovascular Events
}

\author{
Marc S. Sabatine, M.D., M.P.H., Robert P. Giugliano, M.D., \\ Stephen D. Wiviott, M.D., Frederick J. Raal, M.B., B.Ch., M.Med., Ph.D., \\ Dirk J. Blom, M.B., Ch.B., M.Med., Ph.D., Jennifer Robinson, M.D., M.P.H., \\ Christie M. Ballantyne, M.D., Ransi Somaratne, M.D., Jason Legg, Ph.D., \\ Scott M. Wasserman, M.D., Robert Scott, M.D., Michael J. Koren, M.D., \\ and Evan A. Stein, M.D., Ph.D., for the Open-Label Study of Long-Term \\ Evaluation against LDL Cholesterol (OSLER) Investigators
}

\section{A BSTRACT}

\section{BACKGROUND}

Evolocumab, a monoclonal antibody that inhibits proprotein convertase subtilisinkexin type 9 (PCSK9), significantly reduced low-density lipoprotein (LDL) cholesterol levels in short-term studies. We conducted two extension studies to obtain longer-term data.

\section{METHODS}

In two open-label, randomized trials, we enrolled 4465 patients who had completed 1 of 12 phase 2 or 3 studies ("parent trials") of evolocumab. Regardless of studygroup assignments in the parent trials, eligible patients were randomly assigned in a 2:1 ratio to receive either evolocumab ( $140 \mathrm{mg}$ every 2 weeks or $420 \mathrm{mg}$ monthly) plus standard therapy or standard therapy alone. Patients were followed for a median of 11.1 months with assessment of lipid levels, safety, and (as a prespecified exploratory analysis) adjudicated cardiovascular events including death, myocardial infarction, unstable angina, coronary revascularization, stroke, transient ischemic attack, and heart failure. Data from the two trials were combined.

\section{RESULTS}

As compared with standard therapy alone, evolocumab reduced the level of LDL cholesterol by $61 \%$, from a median of $120 \mathrm{mg}$ per deciliter to $48 \mathrm{mg}$ per deciliter $(\mathrm{P}<0.001)$. Most adverse events occurred with similar frequency in the two groups, although neurocognitive events were reported more frequently in the evolocumab group. The risk of adverse events, including neurocognitive events, did not vary significantly according to the achieved level of LDL cholesterol. The rate of cardiovascular events at 1 year was reduced from $2.18 \%$ in the standard-therapy group to $0.95 \%$ in the evolocumab group (hazard ratio in the evolocumab group, $0.47 ; 95 \%$ confidence interval, 0.28 to $0.78 ; \mathrm{P}=0.003$ ).

\section{CONCLUSIONS}

During approximately 1 year of therapy, the use of evolocumab plus standard therapy, as compared with standard therapy alone, significantly reduced LDL cholesterol levels and reduced the incidence of cardiovascular events in a prespecified but exploratory analysis. (Funded by Amgen; OSLER-1 and OSLER-2 ClinicalTrials.gov numbers, NCT01439880 and NCT01854918.)
From the Thrombolysis in Myocardial Infarction (TIMI) Study Group, Division of Cardiovascular Medicine, Brigham and Women's Hospital, and the Department of Medicine, Harvard Medical School, Boston (M.S.S., R.P.G., S.D.W.); the Carbohydrate and Lipid Metabolism Research Unit, Faculty of Health Sciences, University of the Witwatersrand, Johannesburg (F.J.R.), and the Division of Lipidology, Department of Medicine, University of Cape Town, Cape Town (D.J.B.) - both in South Africa; the Departments of Epidemiology and Medicine, College of Public Health, University of lowa, lowa City (J.R.); the Sections of Cardiovascular Research and Cardiology, Department of Medicine, Baylor College of Medicine, and the Center for Cardiovascular Disease Prevention, Houston Methodist DeBakey Heart and Vascular Center, Houston (C.M.B.); Amgen, Thousand Oaks, CA (R. Somaratne, J.L., S.M.W., R. Scott); Jacksonville Center for Clinical Research, Jacksonville, FL (M.J.K.); and the Metabolic and Atherosclerosis Research Center, Cincinnati (E.A.S.). Address reprint requests to Dr. Sabatine at the TIMI Study Group, Cardiovascular Division, Brigham and Women's Hospital, 75 Francis St., Boston, MA 02115, or at msabatine@ partners.org; or to Dr. Stein at the Metabolic and Atherosclerosis Research Center, 5355 Medpace Way, Cincinnati, $\mathrm{OH}$ 45225, or at esteinmrl@aol.com.

This article was published on March 15, 2015, at NEJM.org.

DOI: 10.1056/NEJMoal500858

Copyright $\odot 2015$ Massachusetts Medical Society. 
EDUCTION IN LOW-DENSITY LIPOPROtein (LDL) cholesterol levels has proved to be highly effective in reducing rates of major cardiovascular events in numerous large outcome trials. ${ }^{1-3}$ For this reason, LDL cholesterol reduction has been incorporated into practice guidelines as a fundamental means of reducing cardiovascular morbidity and mortality. ${ }^{4-7}$

During the past 3 years, monoclonal antibodies that inhibit proprotein convertase subtilisinkexin type 9 (PCSK9) have emerged as a new class of drugs that very effectively lower LDL cholesterol levels. ${ }^{8}$ One of the members of this class is evolocumab, a fully human monoclonal antibody that typically achieves approximately a $60 \%$ reduction in $\mathrm{LDL}$ cholesterol levels when administered at the doses that were studied in phase 3 trials. ${ }^{9-13}$

On completing a trial of evolocumab (parent trial), patients could enroll into one of two longer-term extension trials, designated Open-Label Study of Long-Term Evaluation against LDL Cholesterol 1 (OSLER-1), for patients completing phase 2 trials, and OSLER-2, for those completing phase 3 trials. The OSLER-1 and OSLER-2 trials had as their primary goal the gathering of longer-term data on safety, side-effect profile, and LDL cholesterol reduction and also included a prespecified exploratory analysis on adjudicated cardiovascular outcomes. Here, we report on the combined results of the OSLER- 1 and OSLER-2 trials.

\section{METHODS}

\section{STUDY DESIGN AND OVERSIGHT}

The OSLER-1 trial was an open-label, randomized, controlled study conducted at 190 centers that participated in at least one of five phase 2 studies of evolocumab. ${ }^{14-19}$ Analogously, the OSLER-2 trial was an open-label, randomized, controlled study conducted at 305 centers that participated in at least one of seven phase 3 studies of evolocumab (Table 1). ${ }^{9-13,20,21}$

The protocols for the OSLER- 1 and OSLER-2 trials were approved by the relevant ethics committee at each participating site and are available with the full text of this article at NEJM.org. Amgen sponsored and designed the two trials and was responsible for data collection and analysis. The first draft of the manuscript was written by the first and last authors. All the coauthors participated in subsequent revisions of the manuscript. The academic authors had full access to the data and vouch for their accuracy and completeness and for the analyses as presented and for the fidelity of this report to the trial protocols. The first and last authors made the decision to submit the manuscript for publication.

\section{PATIENTS}

There was variation in the clinical characteristics of the patients enrolled in the 12 parent studies (Table 1). Patients who had completed one of the parent studies could enroll in one of the OSLER extension studies, provided that they did not have an adverse event that led to the discontinuation of a study drug during the parent trial, did not have an unstable medical condition (in the judgment of the investigator), and were not expected to need unblinded lipid measurements or adjustment of background lipid-regulating therapy during the first 12 weeks of participation in the OSLER trials. All patients provided written informed consent before enrollment in the extension study.

\section{RANDOMIZATION, STUDY TREATMENT,} AND FOLLOW-UP

Regardless of the study-group assignment in the parent study, eligible patients were randomly assigned at the last visit in the parent study, or as soon as possible thereafter, to receive either evolocumab plus standard therapy (evolocumab group) or standard therapy alone (standard-therapy group) in a 2:1 ratio. Randomization was performed centrally with the use of an interactive voice-response or Web-response system, with stratification in OSLER-1 according to the study-group assignment in the parent trial and in OSLER-2 according to the parent trial and study-drug dose frequency in the parent trial.

Evolocumab was administered subcutaneously at a dose of $420 \mathrm{mg}$ once a month in OSLER-1 and, on the basis of patient choice, at a dose of either $140 \mathrm{mg}$ every 2 weeks or $420 \mathrm{mg}$ once a month in OSLER-2. Both regimens have been shown to reduce LDL cholesterol levels by approximately $60 \%$ in this patient population..$^{9-13}$ All patients, investigators, and care providers were aware of the randomized treatment assignments; no placebo was used for the standard-therapy group. Standard-of-care background therapy in the two groups was based on local guidelines for the treatment of LDL cholesterol. 


\begin{tabular}{|c|c|c|c|}
\hline Trial & $\begin{array}{l}\text { No. of Patients } \\
\text { Enrolled in } \\
\text { Parent Trial'i }\end{array}$ & $\begin{array}{l}\text { No. of Patients } \\
\text { Enrolled in } \\
\text { OSLER Trials }\end{array}$ & Patient Population \\
\hline \multicolumn{4}{|l|}{ Phase $2 \div$} \\
\hline MENDEL-1 ${ }^{15}$ & 406 & 300 & $\begin{array}{l}\text { LDL cholesterol level, } \geq 100 \text { and }<190 \mathrm{mg} / \mathrm{dl} \text {; no background antilipid } \\
\text { therapy }\end{array}$ \\
\hline LAPLACE-TIMI $57^{14}$ & 629 & 530 & $\begin{array}{l}\text { LDL cholesterol level, } \geq 85 \mathrm{mg} / \mathrm{dl} \text { while receiving statin with or with- } \\
\text { out ezetimibe }\end{array}$ \\
\hline GAUSS-1 $1^{17}$ & 157 & 128 & Statin-intolerant; LDL cholesterol level, $\geq 100 \mathrm{mg} / \mathrm{dl}$ \\
\hline RUTHERFORD- $1^{16}$ & 167 & 147 & $\begin{array}{l}\text { Heterozygous familial hypercholesterolemia; LDL cholesterol level, } \\
\geq 100 \mathrm{mg} / \mathrm{dl} \text { while receiving statin with or without ezetimibe }\end{array}$ \\
\hline YUKAWA-1 ${ }^{19}$ & 307 & 219 & $\begin{array}{l}\text { High-risk patients in Japan; LDL cholesterol level, } \geq 116 \mathrm{mg} / \mathrm{dl} \text { while } \\
\text { receiving statin }\end{array}$ \\
\hline \multicolumn{4}{|l|}{ Phase 3j } \\
\hline MENDEL-2 ${ }^{11}$ & 614 & 378 & $\begin{array}{l}\text { LDL cholesterol level, } \geq 100 \text { and }<190 \mathrm{mg} / \mathrm{dl} \text {; no background anti- } \\
\text { lipid therapy }\end{array}$ \\
\hline LAPLACE-2 ${ }^{10}$ & 1896 & 1382 & $\begin{array}{l}\text { LDL cholesterol level, } \geq 80 \mathrm{mg} / \mathrm{dl} \text { (intensive statin at screening), } \\
\geq 100 \mathrm{mg} / \mathrm{dl} \text { (nonintensive statin at screening), or } \geq 150 \mathrm{mg} / \mathrm{dl} \\
\text { (no statin at screening); moderate- or high-intensity statin with } \\
\text { or without ezetimibe }\end{array}$ \\
\hline GAUSS-2 ${ }^{12}$ & 307 & 254 & Statin-intolerant patients; LDL cholesterol level, $\geq 100$ and $<190 \mathrm{mg} / \mathrm{dl}$ \\
\hline RUTHERFORD- $2^{13}$ & 329 & 293 & $\begin{array}{l}\text { Heterozygous familial hypercholesterolemia; LDL cholesterol level, } \\
\quad \geq 100 \mathrm{mg} / \mathrm{dl} \text { while receiving statin with or without ezetimibe }\end{array}$ \\
\hline DESCARTES ${ }^{9}$ & 901 & 612 & $\begin{array}{l}\text { LDL cholesterol level, } \geq 75 \mathrm{mg} / \mathrm{dl} \text { while receiving statin with or with- } \\
\text { out ezetimibe }\end{array}$ \\
\hline THOMAS-1 ${ }^{20}$ & 149 & 112 & LDL cholesterol level, $>85 \mathrm{mg} / \mathrm{dl}$ while receiving statin \\
\hline THOMAS-2 $2^{21}$ & 164 & 110 & LDL cholesterol level, $>85 \mathrm{mg} / \mathrm{dl}$ while receiving statin \\
\hline \multicolumn{4}{|c|}{$\begin{array}{l}\text { To convert the values for low-density lipoprotein (LDL) cholesterol to millimoles per liter, multiply by } 0.02586 \text {. } \\
\text { Included in this category is the number of patients who underwent randomization and received at least one dose of a study drug. } \\
\text { Patients who were enrolled in phase } 2 \text { parent trials and who agreed to participate in the extension study were enrolled in OSLER-1. } \\
\text { Patients who were enrolled in phase } 3 \text { parent trials and who agreed to participate in the extension study were enrolled in OSLER-2. }\end{array}$} \\
\hline
\end{tabular}

To avoid inadvertent unblinding of the parent study, lipid results from the central laboratory were unblinded only after the week 12 visit in the OSLER trials. Adjustments to background lipidlowering therapies were discouraged. The studyvisit schedules were similar in OSLER-1 and OSLER-2. Common to the two trials, patients were to have in-person clinic visits on day 1 and then quarterly at weeks $12,24,36$, and 48 . At other time points, patients in the evolocumab group had inperson visits, whereas patients in the standardtherapy group had telephone contact only.

The trial protocols specified that randomized treatment was to conclude at week 56 in OSLER-1 and at week 48 in OSLER-2. After the end of randomized treatment, all patients were to receive open-label evolocumab for longer-term, nonrandomized assessment of efficacy and safety.

\section{END POINTS}

The primary end point in the two trials was the incidence of adverse events. Additional safety end points included serious adverse events, adverse events leading to the discontinuation of the study drug (for patients in the evolocumab group), abnormalities in creatine kinase levels and liverfunction testing, and the development of binding and neutralizing antibodies against evolocumab, which were assayed as reported previously. ${ }^{22}$

The secondary end point was the percent change in the LDL cholesterol level. Other efficacy lipid measurements included non-high-density lipoprotein (HDL) cholesterol, total cholesterol, triglycerides, HDL cholesterol, apolipoproteins A1 and $\mathrm{B}$, and lipoprotein(a). Lipids were measured at a central laboratory (Medpace Reference Laboratories, Cincinnati, and Leuven, Belgium) after 
a fast of at least 9 hours. The LDL cholesterol level was calculated with the use of the Friedewald formula. ${ }^{23}$

A prespecified exploratory outcome was the incidence of adjudicated cardiovascular events, which was ascertained over the course of the study. (Definitions are provided in the Methods section in the Supplementary Appendix, available at NEJM.org.) Cardiovascular events included death, coronary events (myocardial infarction, unstable angina requiring hospitalization, or coronary revascularization), cerebrovascular events (stroke or transient ischemic attack), and heart failure requiring hospitalization. Potential cardiovascular events were adjudicated by the central clinical-events committee at the Thrombolysis in Myocardial Infarction (TIMI) Study Group in Boston, whose members were unaware of treatment assignments. All cardiovascular events were combined in an exploratory composite analysis that was based on the events that were prespecified in the trial protocols. In addition, all cardiovascular end points except for heart failure were combined into a post hoc composite of major adverse cardiovascular events.

\section{STATISTICAL ANALYSIS}

The timing of the analysis in this report was triggered by a planned submission of a biologics license application to the Food and Drug Administration. The authors decided to present these data to the scientific community at the next major scientific meeting. In anticipation of that meeting, the data were updated with cardiovascular outcomes that were based on adjudicated data through January 21, 2015, along with demographic, lipid, and safety data that were based on cleaned data through October 31, 2014.

The data from the OSLER- 1 and OSLER-2 trials were combined into a single analysis set. Data for patients were censored at the start of the uncontrolled period in each study. Lipid measurements were summarized with the use of means or medians, and treatment differences were tested with the use of the Wilcoxon rank-sum test without adjustment for multiplicity. We used the Kaplan-Meier method to estimate time-to-event cumulative incidence on an intention-to-treat basis. Safety was described according to the incidence of adverse events. The minimum LDL cholesterol category for a patient was determined by the minimum value observed in the randomized, con- trolled period of the studies. We used the log-rank test to analyze the difference in cumulative incidence curves for cardiovascular events. Hazard ratios were estimated with the use of Cox proportional-hazard models without stratification. Statistical analyses were performed with the use of SAS software, version 9.3, and $\mathrm{R}$ software, version 3.0.3.

\section{RESULTS}

\section{PATIENTS}

From October 2011 through June 2014, we enrolled a total of 4465 patients in the OSLER program (1324 patients in OSLER-1 and 3141 patients in OSLER-2), which represented $74.1 \%$ of eligible patients in the parent studies (Table 1). Of the 4465 patients, 2976 were randomly assigned to receive evolocumab plus standard therapy and 1489 to receive standard therapy alone (Fig. S1 in the Supplementary Appendix). The median duration of follow-up was 11.1 months (interquartile range, 11.0 to 12.8), for a total of 4219.4 patient-years of follow-up.

The mean age of the patients was 58 years, and $80.4 \%$ had at least one cardiovascular risk factor (Table 2). A total of 3128 patients (70.1\%) were receiving statin therapy at the start of the OSLER trials. Premature permanent discontinuation of evolocumab occurred in $7.2 \%$ of patients. Changes in open-label statin therapy were infrequent (for details, see the Results section in the Supplementary Appendix).

\section{LIPID CHANGES}

The median baseline LDL cholesterol, before randomization into a parent study, was $120 \mathrm{mg}$ per deciliter. At the week 12 visit in the OSLER trials, evolocumab, as compared with standard therapy, reduced the LDL cholesterol level by 61\% (95\% confidence interval [CI], 59 to 63 ; $\mathrm{P}<0.001$ ), for a mean absolute reduction of $73 \mathrm{mg}$ per deciliter to a median of $48 \mathrm{mg}$ per decileter. Data were similar in the OSLER-1 and OSLER-2 trials (Table S1 in the Supplementary Appendix). The reduction in LDL cholesterol levels with evolocumab was consistent over time (Fig. 1). At 12 weeks, the LDL cholesterol level was reduced to $100 \mathrm{mg}$ per deciliter or less in $90.2 \%$ of patients and to $70 \mathrm{mg}$ per deciliter or less in $73.6 \%$ of patients in the evolocumab group, as compared with $26.0 \%$ and $3.8 \%$, respectively, in the standard-therapy group. 


\begin{tabular}{|c|c|c|}
\hline Characteristic & $\begin{array}{c}\text { Evolocumab } \\
\text { Group } \\
(\mathrm{N}=\mathbf{2 9 7 6})\end{array}$ & $\begin{array}{c}\text { Standard-Therapy } \\
\text { Group } \\
(\mathrm{N}=1489)\end{array}$ \\
\hline Mean age $\pm S D-y r$ & $57.8 \pm 11.0$ & $58.2 \pm 10.9$ \\
\hline Male sex — no. (\%) & $1490(50.1)$ & $765(51.4)$ \\
\hline White race - no. $(\%) \dagger$ & $2559(86.0)$ & $1267(85.1)$ \\
\hline \multicolumn{3}{|l|}{ Region } \\
\hline North America & $1402(47.1)$ & $705(47.3)$ \\
\hline Europe & $1205(40.5)$ & $597(40.1)$ \\
\hline Asia Pacific or South Africa & $369(12.4)$ & $187(12.6)$ \\
\hline Cardiovascular risk factor — no. (\%) & $2379(79.9)$ & $1211(81.3)$ \\
\hline Hypertension & $1545(51.9)$ & $777(52.2)$ \\
\hline Diabetes mellitus & $382(12.8)$ & $217(14.6)$ \\
\hline Metabolic syndrome & $1035(34.8)$ & 475 (31.9) \\
\hline Current cigarette use & $465(15.6)$ & $222(14.9)$ \\
\hline Family history of premature coronary artery disease $\downarrow$ & $724(24.3)$ & $362(24.3)$ \\
\hline Known familial hypercholesterolemia & $289(9.7)$ & $151(10.1)$ \\
\hline Moderately high risk or high risk on NCEPJ & $1332(44.8)$ & $693(46.5)$ \\
\hline \multicolumn{3}{|l|}{ Coronary artery disease — no. (\%) } \\
\hline Any & $589(19.8)$ & $307(20.6)$ \\
\hline Myocardial infarction & $276(9.3)$ & $141(9.5)$ \\
\hline Percutaneous coronary intervention & $325(10.9)$ & $170(11.4)$ \\
\hline Coronary-artery bypass grafting & $185(6.2)$ & $110(7.4)$ \\
\hline \multicolumn{3}{|l|}{ Cerebrovascular or peripheral-artery disease — no. (\%) } \\
\hline Any & $266(8.9)$ & $141(9.5)$ \\
\hline Carotid- or vertebral-artery disease & $94(3.2)$ & $62(4.2)$ \\
\hline Stroke & $81(2.7)$ & $37(2.5)$ \\
\hline Peripheral-artery disease & $85(2.9)$ & $50(3.4)$ \\
\hline \multicolumn{3}{|l|}{ Medication use - no. (\%) } \\
\hline \multicolumn{3}{|l|}{ Statin } \\
\hline Any & $2073(69.7)$ & $1055(70.9)$ \\
\hline High-intensity & $795(26.7)$ & $415(27.9)$ \\
\hline Moderate-intensity & $1034(34.7)$ & $522(35.1)$ \\
\hline Low-intensity & $240(8.1)$ & $118(7.9)$ \\
\hline Unknown & $4(0.1)$ & 0 \\
\hline Ezetimibe & $376(12.6)$ & $229(15.4)$ \\
\hline \multicolumn{3}{|c|}{ Median lipid measure at baseline in parent study (IQR) $-\mathrm{mg} / \mathrm{dl}$} \\
\hline LDL cholesterol & $120(97-148)$ & $121(97-151)$ \\
\hline Total cholesterol & $202(175-234)$ & $205(174-235)$ \\
\hline HDL cholesterol & $51(42-62)$ & $51(42-62)$ \\
\hline Triglycerides & $120(89-165)$ & $119(89-167)$ \\
\hline
\end{tabular}

* Baseline characteristics are based on data that were obtained at the start of the parent study; values for statin use and intensity are based on data from the start of the OSLER program. There were no significant differences between the two groups at baseline. To convert the values for triglycerides to millimoles per liter, multiply by 0.01129 . HDL denotes high-density lipoprotein, and IQR interquartile range.

$\uparrow$ Race was self-reported.

t A family history of premature coronary artery disease was defined as the presence of coronary artery disease in a firstdegree male relative 55 years of age or younger or in a first-degree female relative 65 years of age or younger.

$\int$ According to the criteria of the National Cholesterol Education Program (NCEP), moderately high or high risk is defined as coronary heart disease, coronary heart disease equivalent, or two or more cardiovascular risk factors with an estimated 10-year risk of cardiovascular events of $10 \%$ or more.

q The intensity of statin therapy was defined according to recent guidelines. ${ }^{4}$

The New England Journal of Medicine 


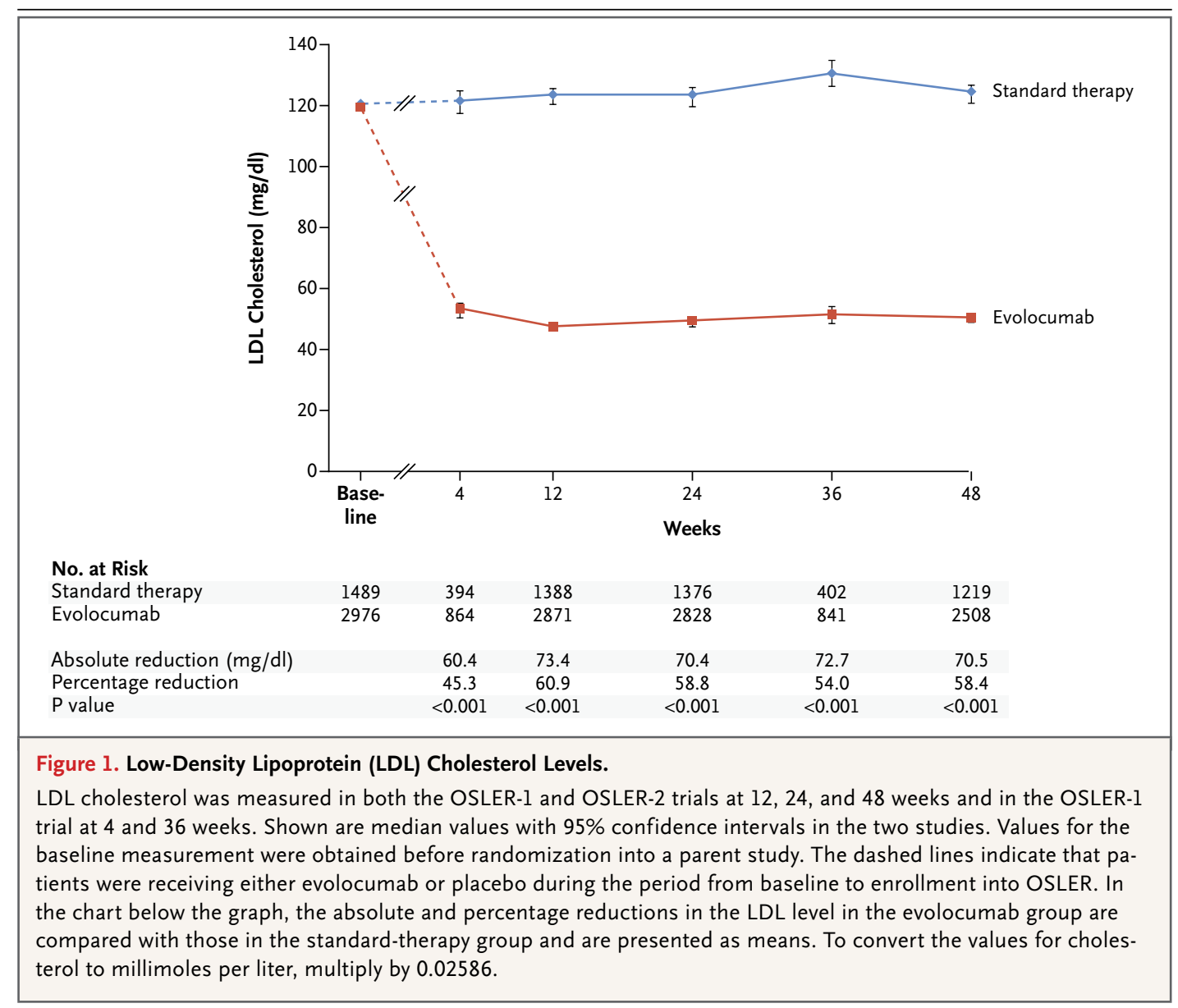

In the evolocumab group, as compared with the standard-therapy group, changes in related atherogenic lipid measures were similar to those observed for LDL cholesterol, with reductions of $52.0 \%$ in non-HDL cholesterol, $47.3 \%$ in apolipoprotein B, 36.1\% in total cholesterol, $12.6 \%$ in triglycerides, and $25.5 \%$ in lipoprotein(a) $(\mathrm{P}<0.001$ for all comparisons) (Fig. S2 in the Supplementary Appendix). Evolocumab raised levels of HDL cholesterol and apolipoprotein A1 by 7.0\% and 4.2\%, respectively ( $\mathrm{P}<0.001$ for both comparisons).

\section{SAFETY AND SIDE-EFFECT PROFILE}

Adverse events occurred in 2060 of 2976 patients (69.2\%) in the evolocumab group and in 965 of 1489 patients $(64.8 \%)$ in the standard-therapy group (Table 3). Serious adverse events occurred in 222 patients $(7.5 \%)$ in the evolocumab group and in 111 patients (7.5\%) in the standard-therapy group. Elevations in aminotransferase or creatine kinase levels occurred at a similar rate in the two groups: $1.0 \%$ in the evolocumab group and $1.2 \%$ in the standard-therapy group for elevated amino transferase levels and $0.6 \%$ and $1.1 \%$, respectively, for elevated creatine kinase levels. Although the rate of neurocognitive adverse events was low $(<1 \%)$, such events were reported more frequently in the evolocumab group. Of note, the incidence of neurocognitive adverse events did not appear to be related to the LDL cholesterol level during treatment (Table S2 in the Supplementary Appendix). Other adverse events are listed in Table S3 in the Supplementary Appendix.

Injection-site reactions were reported in 129 patients (4.3\%) in the evolocumab group (the only group in which such events were analyzed) and led to discontinuation of evolocumab in $6 \mathrm{pa}-$ tients $(0.2 \%)$. New evolocumab-binding antibodies were detected in 9 patients $(0.3 \%)$ in the evolocumab group and in 4 patients $(0.3 \%)$ in the standard-therapy group, and binding-antibody titers were transient in patients who had repeat testing. No neutralizing antibodies against evolocumab were detected. 


\begin{tabular}{|c|c|c|}
\hline \multirow[t]{2}{*}{ Variable } & $\begin{array}{l}\text { Evolocumab Group } \\
\qquad(N=2976)\end{array}$ & $\begin{array}{l}\text { Standard-Therapy Group } \\
\qquad(\mathrm{N}=1489)\end{array}$ \\
\hline & \multicolumn{2}{|c|}{ no. (\%) } \\
\hline \multicolumn{3}{|l|}{ Adverse events } \\
\hline Any & $2060(69.2)$ & $965(64.8)$ \\
\hline Serious & $222(7.5)$ & $111(7.5)$ \\
\hline Leading to discontinuation of evolocumab & $71(2.4)$ & NA \\
\hline Muscle-related & $190(6.4)$ & $90(6.0)$ \\
\hline Injection-site reaction & $129(4.3)$ & NA \\
\hline Neurocognitive event $†$ & $27(0.9)$ & $4(0.3)$ \\
\hline \multicolumn{3}{|l|}{ Othert } \\
\hline Arthralgia & $137(4.6)$ & $48(3.2)$ \\
\hline Headache & $106(3.6)$ & $32(2.1)$ \\
\hline Limb pain & $99(3.3)$ & $32(2.1)$ \\
\hline Fatigue & $83(2.8)$ & $15(1.0)$ \\
\hline \multicolumn{3}{|l|}{ Laboratory results } \\
\hline $\begin{array}{l}\text { Alanine or aspartate aminotransferase }>3 \times U L N \text { at any visit after } \\
\text { baseline }\end{array}$ & $31(1.0)$ & $18(1.2)$ \\
\hline Creatine kinase $>5 \times U L N$ at any visit after baseline & $17(0.6)$ & $17(1.1)$ \\
\hline \multicolumn{3}{|c|}{$\begin{array}{l}* \text { NA denotes not applicable, and ULN upper limit of the normal range. } \\
\text { T Neurocognitive events were delirium (including confusion), cognitive and attention disorders and disturbances, dementia } \\
\text { and amnestic conditions, disturbances in thinking and perception, and mental impairment disorders. } \\
+ \text { Included in this category are adverse events that were reported in at least } 1 \% \text { of patients in the evolocumab group and } \\
\text { in more patients in the evolocumab group than in the standard-therapy group by at least } 1 \text { percentage point. }\end{array}$} \\
\hline
\end{tabular}

Rates of overall adverse events, serious adverse events, and elevations in aminotransferase or creatine kinase levels were similar among patients in the evolocumab group who had LDL cholesterol levels of less than $40 \mathrm{mg}$ per deciliter or less than $25 \mathrm{mg}$ per deciliter as in those with higher levels during OSLER (Table S2 in the Supplementary Appendix).

\section{CARDIOVASCULAR EVENTS}

Cardiovascular events were prospectively adjudicated in an exploratory analysis (Table S4 in the Supplementary Appendix). When these events were combined in a composite of all cardiovascular events, patients in the evolocumab group had a significantly lower rate of all cardiovascular events than did patients in the standard-therapy group (Kaplan-Meier estimates at 1 year, $0.95 \%$ and $2.18 \%$, respectively; hazard ratio, $0.47 ; 95 \%$ CI, 0.28 to $0.78 ; \mathrm{P}=0.003$ ). The cumulative incidence curves diverged progressively over time (Fig. 2). Similar results were obtained for the post hoc composite of major adverse cardiovascular events (Table S4 in the Supplementary Appendix).

\section{DISCUSSION}

In the OSLER-1 and OSLER-2 trials, open-label treatment with evolocumab reduced LDL cholesterol levels by $61 \%$, from a pretreatment median level of $120 \mathrm{mg}$ per deciliter to a 12-week ontreatment median level of $48 \mathrm{mg}$ per deciliter. This result was consistent with reports from previous short-term trials of evolocumab. ${ }^{9-17,19}$ In the OSLER trials, the reduction in LDL cholesterol levels was sustained through 48 weeks, a finding consistent with the results of a much smaller study of evolocumab. ${ }^{9}$ Effects on other lipid fractions were also similar to those seen in previous studies.

Some nonspecific adverse events (arthralgia, headache, limb pain, and fatigue) and neurocognitive adverse events were reported more frequently in the evolocumab group than in the 


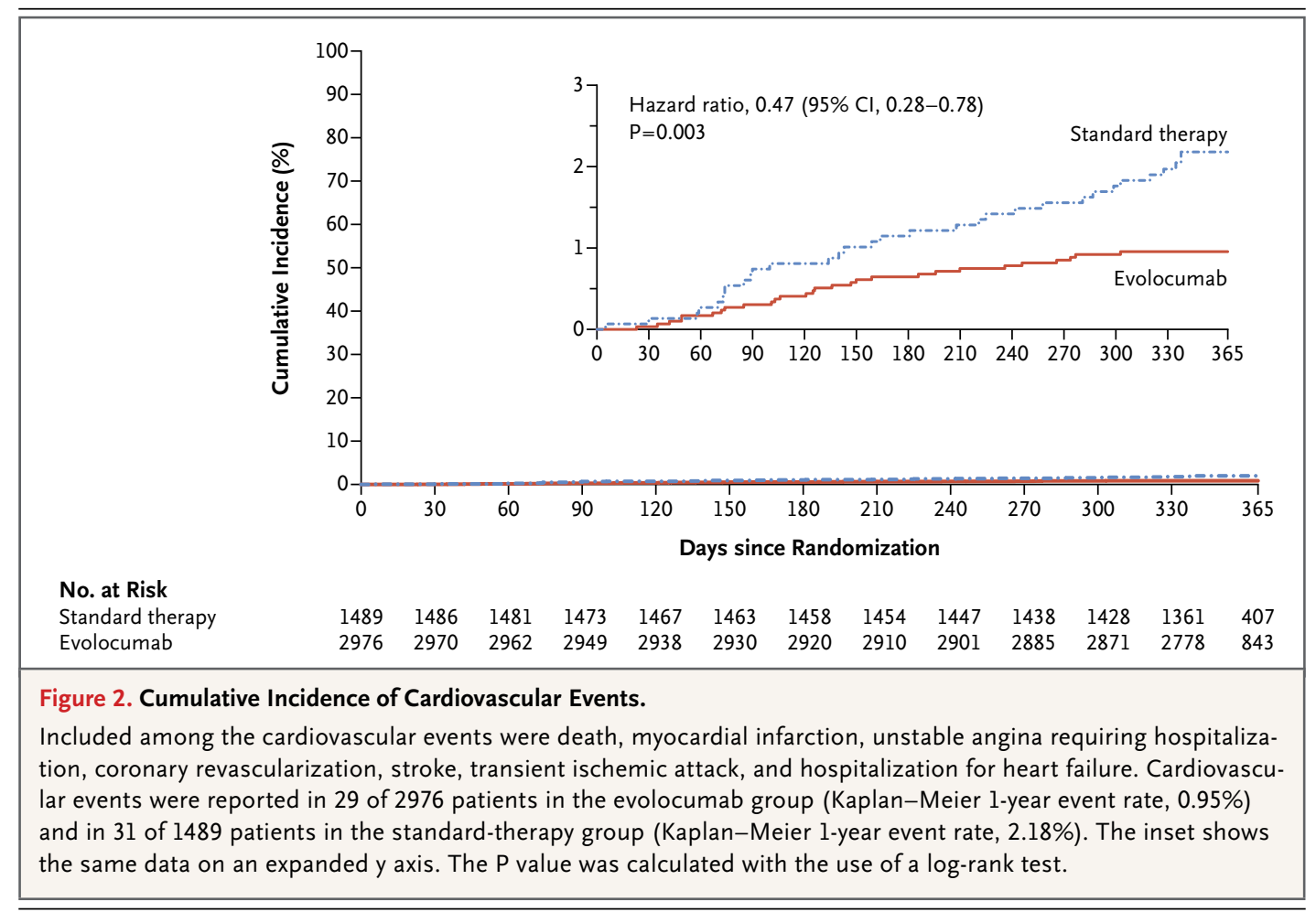

standard-therapy group. These observations should be viewed in the context of the greater number of protocol-stipulated in-person visits for patients in the evolocumab group as well as the open-label nature of the trial, which could predispose patients who are receiving the study intervention to be more likely to note adverse events, particularly those associated with other therapies that reduce LDL cholesterol. ${ }^{24}$ Moreover, there did not appear to be any excess of these events in patients who had very low versus higher on-treatment levels of LDL cholesterol. Furthermore, PCSK9 loss-of-function variants have not been associated with impaired cognitive performance. ${ }^{25}$ Nonetheless, much larger trials, including a dedicated neurocognitive substudy (ClinicalTrials.gov number, NCT02207634), ${ }^{26}$ are ongoing to provide more definitive assessments of safety over longer-term follow-up.

An unanswered question is whether a reduction in the LDL cholesterol level with a PCSK9 inhibitor will lead to a reduction in cardiovascular events. Reduction in such events with statins has been well established during the past two decades in numerous large, randomized, controlled trials. ${ }^{1,2}$ In contrast, it has been difficult to show that adding other lipid-modifying drugs to statins results in a further decrease in cardiovascular events, which is probably due to the modest effects on LDL cholesterol or off-target adverse effects. ${ }^{27-30}$ This issue was a factor contributing to the most recent set of practice guidelines from the American College of CardiologyAmerican Heart Association, in which the role of nonstatin lipid-modifying drugs was diminished, as compared with previous guidelines. ${ }^{4}$ Recently, however, in the Improved Reduction of Outcomes: Vytorin Efficacy International Trial (IMPROVE-IT), an additional reduction in LDL cholesterol levels with the addition of ezetimibe, a cholesterol-absorption inhibitor, to a statin significantly reduced cardiovascular events, as compared with statin monotherapy. ${ }^{3}$ These data have refocused attention on the potential cardiovascular benefit of greater LDL cholesterol reduction through nonstatin mechanisms.

Evolocumab, unlike other nonstatin lipidmodifying drugs, reduces LDL cholesterol levels as much as, if not more than, high doses of statins. Also, PCSK9 inhibitors have the same ultimate mechanism for LDL reduction as statins - namely, by increasing LDL receptor activity on 
the hepatocyte surface, ${ }^{8}$ suggesting that PCSK9 inhibitors should similarly have a beneficial effect on cardiovascular outcomes. Additional support for the potential of PCSK9 inhibition in reducing cardiovascular events comes from the observation that loss-of-function genetic variants leading to reductions in PCSK9 activity have been associated with significantly lower lifetime rates of cardiovascular events. ${ }^{31}$ Thus, the reduction in cardiovascular events seen in the OSLER trials within the first year of evolocumab therapy, even though the analysis was both exploratory and based on a relatively small number of events, is consistent with the large reduction in LDL cholesterol levels, the mechanism of action, and the PCSK9 genetic data.

The Further Cardiovascular Outcomes Research with PCSK9 Inhibition in Subjects with Elevated Risk (FOURIER) study (NCT01764633) is an ongoing trial that is intended to provide a definitive assessment of the cardiovascular benefit of evolocumab. The FOURIER study is a randomized, placebo-controlled trial involving 27,500 high-risk patients with cardiovascular disease who are receiving background statin therapy; the primary end point is a composite of cardiovascular death, myocardial infarction, hospitalization for unstable angina, stroke, or coronary revascularization. ${ }^{32}$ Similar trials of alirocumab and bococizumab to evaluate cardiovascular outcomes are in progress (NCT01663402, NCT01975376, and NCT01975389). ${ }^{33-35}$

Several study limitations are noteworthy. First, the open-label design of the trials could have had an influence on the reporting of events, both cardiovascular and safety. This issue would es- pecially be a concern for coronary revascularization, the single most frequently reported cardiovascular event, since the decision to perform this procedure could have been influenced by knowledge of treatment assignment. Second, the numbers of cardiovascular and select adverse events were relatively small. Third, although rates of adverse events and study-drug discontinuation were low in the parent trials, ${ }^{9-17,19}$ patients were eligible to transition to the OSLER trials if they had not had an adverse event that led to the discontinuation of a study drug. Thus, data on safety and side-effect profiles in our study come from a cohort of patients who had all successfully received injections and many of whom had received evolocumab for at least 12 weeks. Fourth, the OSLER program included a mix of patients with varying degrees of cardiovascular risk and use and intensity of statin therapy. Thus, not all the study patients would necessarily have been the optimal target population for this novel treatment.

In conclusion, patients who had previously participated in 12 shorter-term parent trials of the PCSK9 inhibitor evolocumab underwent repeat randomization to receive either evolocumab or standard therapy in the OSLER program. Evolucumab reduced levels of LDL cholesterol by $61 \%$ by 12 weeks, with sustained reduction through the median 11-month follow-up. In a prespecified exploratory analysis, there was evidence of a reduction in the rate of cardiovascular events among patients receiving evolocumab.

Supported by Amgen.

Disclosure forms provided by the authors are available with the full text of this article at NEJM.org.

\section{REFERENCES}

1. Baigent C, Keech A, Kearney PM, et al. Efficacy and safety of cholesterol-lowering treatment: prospective meta-analysis of data from 90,056 participants in 14 randomised trials of statins. Lancet 2005; 366:1267-78. [Errata, Lancet 2005;366:1358, 2008;371:2084.]

2. Cholesterol Treatment Trialists' (CTT) Collaboration. Efficacy and safety of more intensive lowering of LDL cholesterol: a meta-analysis of data from 170,000 participants in 26 randomised trials. Lancet 2010;376:1670-81.

3. Cannon CP. IMProved Reduction of Outcomes: Vytorin Efficacy International Trial (IMPROVE-IT). Presented at the American Heart Association Scientific Sessions, Chicago, November 15-18, 2014. abstract.

4. Stone NJ, Robinson JG, Lichtenstein
AH, et al. 2013 ACC/AHA guideline on the treatment of blood cholesterol to reduce atherosclerotic cardiovascular risk in adults: a report of the American College of Cardiology/American Heart Association Task Force on Practice Guidelines. Circulation 2014;129:Suppl 2:S1-S45. [Erratum, Circulation 2014;129:Suppl2:S46-S48.]

5. Reiner Z, Catapano AL, De Backer G, et al. ESC/EAS guidelines for the management of dyslipidaemias: the Task Force for the management of dyslipidaemias of the European Society of Cardiology (ESC) and the European Atherosclerosis Society (EAS). Eur Heart J 2011;32:1769-818.

6. Anderson TJ, Grégoire J, Hegele RA, et al. 2012 Update of the Canadian Cardiovascular Society guidelines for the diagnosis and treatment of dyslipidemia for the prevention of cardiovascular disease in the adult. Can J Cardiol 2013;29:151-67. 7. Jacobson TA, Ito MK, Maki KC, et al. National Lipid Association recommendations for patient-centered management of dyslipidemia: part 1 - executive summary. J Clin Lipidol 2014;8:473-88.

8. Stein EA, Raal FJ. New therapies for reducing low-density lipoprotein cholesterol. Endocrinol Metab Clin North Am 2014;43:1007-33.

9. Blom DJ, Hala T, Bolognese M, et al. A 52-week placebo-controlled trial of evolocumab in hyperlipidemia. N Engl J Med 2014;370:1809-19.

10. Robinson JG, Nedergaard BS, Rogers WJ, et al. Effect of evolocumab or ezetimibe added to moderate- or high-intensity statin therapy on LDL-C lowering in 
patients with hypercholesterolemia: the LAPLACE-2 randomized clinical trial. JAMA 2014;311:1870-82.

11. Koren MJ, Lundqvist P, Bolognese M, et al. Anti-PCSK9 monotherapy for hypercholesterolemia: the MENDEL-2 randomized, controlled phase III clinical trial of evolocumab. J Am Coll Cardiol 2014;63: 2531-40.

12. Stroes E, Colquhoun D, Sullivan D, et al. Anti-PCSK9 antibody effectively lowers cholesterol in patients with statin intolerance: the GAUSS-2 randomized, placebocontrolled phase 3 clinical trial of evolocumab. J Am Coll Cardiol 2014;63: 2541-8.

13. Raal FJ, Stein EA, Dufour R, et al. PCSK9 inhibition with evolocumab (AMG 145) in heterozygous familial hypercholesterolaemia (RUTHERFORD-2): a randomised, double-blind, placebo-controlled trial. Lancet 2015;385:331-40.

14. Giugliano RP, Desai NR, Kohli P, et al. Efficacy, safety, and tolerability of a monoclonal antibody to proprotein convertase subtilisin/kexin type 9 in combination with a statin in patients with hypercholesterolaemia (LAPLACE-TIMI 57): a randomised, placebo-controlled, doseranging, phase 2 study. Lancet 2012;380: 2007-17.

15. Koren MJ, Scott R, Kim JB, et al. Efficacy, safety, and tolerability of a monoclonal antibody to proprotein convertase subtilisin/kexin type 9 as monotherapy in patients with hypercholesterolaemia (MENDEL): a randomised, double-blind, placebo-controlled, phase 2 study. Lancet 2012;380:1995-2006.

16. Raal F, Scott R, Somaratne R, et al. Low-density lipoprotein cholesterol-lowering effects of AMG 145, a monoclonal antibody to proprotein convertase subtilisin/kexin type 9 serine protease in patients with heterozygous familial hypercholesterolemia: the Reduction of LDL-C with PCSK9 Inhibition in Heterozygous Familial Hypercholesterolemia Disorder (RUTHERFORD) randomized trial. Circulation 2012;126:2408-17.

17. Sullivan D, Olsson AG, Scott R, et al. Effect of a monoclonal antibody to PCSK9 on low-density lipoprotein cholesterol levels in statin-intolerant patients: the GAUSS randomized trial. JAMA 2012;308 2497-506.

18. Koren MJ, Giugliano RP, Raal FJ, et al. Efficacy and safety of longer-term administration of evolocumab (AMG 145) in patients with hypercholesterolemia: 52 week results from the Open-Label Study of Long-Term Evaluation Against LDL-C (OSLER) randomized trial. Circulation 2014;129:234-43.

19. Hirayama A, Honarpour N, Yoshida M et al. Effects of evolocumab (AMG 145), a monoclonal antibody to PCSK9, in hypercholesterolemic, statin-treated Japanese patients at high cardiovascular risk-primary results from the phase 2 YUKAWA study. Circ J 2014;78:1073-82.

20. ClinicalTrials.gov. NCT01849497: multi-center, randomized study in subjects with primary hypercholesterolemia or mixed dyslipidemia (http://ClinicalTrials .gov/show/NCT01849497).

21. ClinicalTrials.gov. NCT01879319: randomized, multi-center clinical study in subjects with hypercholesterolemia or mixed dyslipidemia (http://ClinicalTrials .gov/show/NCT01879319).

22. Dias CS, Shaywitz AJ, Wasserman SM et al. Effects of AMG 145 on low-density lipoprotein cholesterol levels: results from 2 randomized, double-blind, placebo-controlled, ascending-dose phase 1 studies in healthy volunteers and hypercholesterolemic subjects on statins. J Am Coll Cardiol 2012;60:1888-98.

23. Friedewald WT, Levy RI, Fredrickson DS. Estimation of the concentration of low-density lipoprotein cholesterol in plasma, without use of the preparative ultracentrifuge. Clin Chem 1972;18:499-502.

24. FDA expands advice on statin risks Silver Spring, MD: Food and Drug Administration (http://www.fda.gov/ForConsumers ConsumerUpdates/ucm293330.htm\#2).

25. Postmus I, Trompet S, de Craen AJ, et al. PCSK9 SNP rs11591147 is associated with low cholesterol levels but not with cognitive performance or noncardiovascular clinical events in an elderly population. J Lipid Res 2013;54:561-6.
26. ClinicalTrials.gov. NCT02207634: Evaluating PCSK9 Binding antiBody Influence oN coGnitive HeAlth in High cardiovascUlar Risk Subjects (EBBINGHAUS) (https://clinicaltrials.gov/ct2/show/ NCT02207634).

27. Barter PJ, Caulfield M, Eriksson $M$, et al. Effects of torcetrapib in patients at high risk for coronary events. N Engl J Med 2007;357:2109-22.

28. Schwartz GG, Olsson AG, Abt M, et al. Effects of dalcetrapib in patients with a recent acute coronary syndrome. $\mathrm{N}$ Engl J Med 2012;367:2089-99.

29. The AIM-HIGH Investigators. Niacin in patients with low HDL cholesterol levels receiving intensive statin therapy. N Engl J Med 2011;365:2255-67. [Erratum, N Engl J Med 2012;367:189.]

30. The HPS2-THRIVE Collaborative Group. Effects of extended-release niacin with laropiprant in high-risk patients. N Engl J Med 2014;371:203-12.

31. Cohen JC, Boerwinkle E, Mosley TH Jr, Hobbs HH. Sequence variations in PCSK9, low LDL, and protection against coronary heart disease. N Engl J Med 2006;354: 1264-72.

32. ClinicalTrials.gov. NCT01764633: Further Cardiovascular Outcomes Research with PCSK9 Inhibition in Subjects with Elevated Risk (FOURIER) (http://ClinicalTrials .gov/show/NCT01764633).

33. ClinicalTrials.gov. NCT01663402: ODYSSEY outcomes: evaluation of cardiovascular outcomes after an acute coronary syndrome during treatment with alirocumab SAR236553 (REGN727) (http:/l ClinicalTrials.gov/show/NCT01663402).

34. ClinicalTrials.gov. NCT01975376: the evaluation of bococizumab (PF-04950615; RN316) in reducing the occurrence of major cardiovascular events in high risk subjects (SPIRE-1) (http://ClinicalTrials.gov/ show/NCT01975376).

35. ClinicalTrials.gov. NCT01975389: the evaluation of bococizumab (PF-04950615; RN316) in reducing the occurrence of major cardiovascular events in high risk subjects (SPIRE-2) (http://ClinicalTrials.gov/ show/NCT01975389).

Copyright (๑) 2015 Massachusetts Medical Society. 